\title{
Onychoscopy: an observational study in 237 patients from the Kashmir Valley of North India
}

\author{
Yasmeen J. Bhat ${ }^{1}$, Muzafar A. Mir ${ }^{1}$, Abid Keen ${ }^{1}$, Iffat Hassan ${ }^{1}$ \\ 1 Postgraduate Department of Dermatology, STD \& Leprosy, Government Medical College, Srinagar, University of Kashmir, Jammu \& \\ Kashmir, India
}

Key words: nail, dermoscopy, onychoscopy, inflammatory nail disorders, melanonychia

Citation: Bhat YJ, Mir MA, Keen A, Hassan I. Onychoscopy: an observational study in 237 patients from the Kashmir Valley of North India. Dermatol Pract Concept. 2018;8(4):283-291. DOI: https://doi.org/10.5826/dpc.0804a06

Received: December 9, 2017; Accepted: April 6, 2018; Published: October 31, 2018

Copyright: $@ 2018$ Bhat et al. This is an open-access article distributed under the terms of the Creative Commons Attribution License, which permits unrestricted use, distribution, and reproduction in any medium, provided the original author and source are credited.

Funding: None.

Competing interests: The authors have no conflicts of interest to disclose.

All authors have contributed significantly to this publication.

Corresponding author: Yasmeen Jabeen Bhat, MD, FACP, Associate Professor, Postgraduate Department of Dermatology, STD \& Leprosy, Government Medical College, Srinagar, University of Kashmir, Jammu \& Kashmir, India-190010. Email: yasmeenasif76@gmail.com

\footnotetext{
ABSTRACT Background: Nail disorders comprise approximately $10 \%$ of all dermatological conditions. Because diagnosis is not always possible by clinical means alone, additional diagnostic procedures may be required at times. Dermoscopy of nails (onychoscopy) has shown promising results in diagnosing various nail disorders and also avoids time-consuming investigations such as culture and biopsy.

Objective: To study the dermoscopic features of various nail disorders to determine the correlation between $\mathrm{KOH}$ examination and onychoscopic patterns in patients with the clinical suspicion of onychomycosis, and to differentiate benign pigmented lesions from malignant ones.

Methods: An open, observational and cross-sectional study of 237 patients was conducted. All patients underwent clinical examination and the affected nails were examined with a dermatoscope. The onychoscopic patterns were identified and recorded.

Results: The study included 237 patients with the following diagnoses: 81 onychomycosis, 63 psoriasis, 27 lichen planus, 30 longitudinal melanonychia, 24 connective tissue disorders, 5 onychophagia and nail tics, 3 subungual verrucae, 2 glomus tumor, 1 Darier disease, and 1 enchondroma. The most common onychoscopic findings were spiked pattern in cases of onychomycosis, dilated and tortuous capillaries in cases of psoriasis, longitudinal streaks and nail fragmentation in cases of lichen planus, and enlarged capillaries in cases of connective tissue diseases.

Limitations: The study was only observational and did not compare the results to biopsy and culture.

Conclusions: Onychoscopy may be used as an important diagnostic tool when evaluating nail disorders. It should be used to aid in the diagnosis of various nail disorders and to avoid unnecessary and time-consuming investigations.
} 


\section{Introduction}

Nail disorders constitute approximately $10 \%$ of all dermatological conditions, and the diagnosis is still a challenge [1]. Keeping in mind the various clinical difficulties in diagnosing nail disorders, and since some dermoscopic patterns are observed consistently with certain diseases, this study was conducted to evaluate various nail changes by using a dermatoscope.

A dermatoscope is a noninvasive diagnostic tool that visualizes subtle clinical patterns of skin lesions, hair disorders, and various nail changes not normally visible to the unaided eye [2].

In addition to diagnosis of common nail disorders, dermoscopy of nails (onychoscopy) provides useful information in the differential diagnosis of longitudinal melanonychia [3-5].The evaluation of longitudinal melanonychia should include personal and family history, clinical examination, and dermoscopic examination. All these together aid in making the differential diagnosis of the nail pigmentation and also help render a decision of whether to biopsy, where to biopsy, and how to biopsy [5,6]. Irregularities in the thickness, parallelism, spacing, and color of the brown-black bands are strongly suggestive of malignant melanoma. These patterns are highly specific and sensitive and may help in the diagnosis, selection of the type of surgery, and management [7].

We studied the dermoscopic features of various nail disorders to determine the correlation between $\mathrm{KOH}$ examination and onychoscopic patterns in patients with clinical diagnosis of onychomycosis and to differentiate various benign and malignant pigmented lesions.

\section{Materials and Methods}

This cross-sectional observational study was conducted in the dermatology outpatient department of a tertiary care hospital. Patients who either presented with nail disorders per se or were having nail involvement associated with various dermatoses were recruited for onychoscopic evaluation over a period of 1 year, from February 2016 through January 2017. Relevant laboratory investigations such as $\mathrm{KOH}$, culture of nail clippings, and nail biopsy were also carried out in selected patients wherever there was diagnostic difficulty. Patients of all age groups who consented to participate were included in the study; there were no exclusion criteria as such. All patients were subjected to clinical examination and dermoscopic examination. In all cases the affected nails were examined by a handheld dermatoscope (DermliteDL3N; 3 Gen, Inc, San Juan Capistrano, CA), with a magnification of $10 \times$. Higher magnification of upto $30 \times$ was used wherever deemed necessary. Images were recorded directly by the digital camera of the dermatoscope with an attachment for iPhone 6. Both nonpolarized and polarized modes were used initially without and then with the fluid. Nonpolarized and dry mode helps in detection of nail plate changes, whereas polarized mode with or without fluid is best for nail bed changes. The linkage fluid used was ultrasound gel in most of the cases. Diagnosis of onychomycosis was made with $\mathrm{KOH}$ examination, and the presence of fungal elements was considered positive for the diagnosis. At times the sample was cultured.

Our study was approved by the institutional ethics committee, and written informed consent was obtained from all patients enrolled in the study.

\section{Results}

A total of 237 patients (140 females and 97 males) were screened for onychoscopic findings. Patients' ages ranged from 1 year to 80 years (mean age: 30.72 years \pm 8.5 ). Most of the patients, that is $151(63.71 \%)$ were from rural areas, whereas $86(36.29 \%)$ patients were from urban areas. Systemic illnesses in the form diabetes mellitus, hypertension, and hypothyroidism were seen in 8,6 , and 2 patients, respectively (not statistically significant).

The various nail disorders in decreasing order of frequency were as follows: onychomycosis (81), psoriasis (63), lichen planus (27), longitudinal melanonychia (30), connective tissue disorders (24), nail tics (5), subungual verrucae (3), glomus tumor (2), Darier disease (1), and calcified enchondroma (1).

The study included $81(34.18 \%)$ patients with clinical suspicion of onychomycosis. Among these patients, 69 had positive direct $\mathrm{KOH}$ examination for onychomycosis and all 12 remaining patients had positive fungal culture. The most common clinical pattern of onychomycosis was distal and lateral subungual onychomycosis and was seen in 62 $(76.54 \%)$ patients, whereas $19(23.46 \%)$ patients had total dystrophic onychomycosis. Onychoscopic findings in these patients showed proximal jagged edge, spikes, and longitudinal striae as shown in Table 1 (Table 1; Figures 1 and 2).

The next common nail disorder was nail psoriasis, which was seen in 63 patients $(28 \%)$. The diagnosis was based solely on clinical examination, and only 1 patient needed nail biopsy. The changes affected both nail plate and nail bed. The nail plate changes observed were pitting that was irregular in size and shape, both longitudinal and transverse ridging and nail plate crumbling. The nail bed changes were onycholysis, which was surrounded by an erythematous border proximally; red to orange salmon patches, which were irregular in size and shape; splinter hemorrhages with color ranging from brown, black, or purple; dilated tortuous capillaries in the hyponychium and proximal nail fold; subungual hyperkeratosis; and pustules in pustular psoriasis (Table 2; Figures 3 and 4). 
TABLE 1. Onychoscopic findings in different types of onychomycosis

\begin{tabular}{|l|c|c|c|c|c|c|}
\hline & \multicolumn{2}{|c|}{ Spiked Pattern } & \multicolumn{2}{c|}{ Longitudinal Striae Pattern } & \multicolumn{2}{c|}{$\begin{array}{c}\text { Distal Irregular } \\
\text { Termination Pattern }\end{array}$} \\
\hline Total & \multicolumn{2}{|c|}{69} & \multicolumn{2}{|c|}{63} & \multicolumn{2}{c|}{33} \\
\hline & No. & $\%$ & No. & \% & No. & 72.73 \\
\hline DLSO & 48 & 69.57 & 45 & 71.43 & 24 & 9 \\
\hline
\end{tabular}

$\mathrm{P}>0.05$; chi-Square statistic $=0.122$

$\mathrm{DLSO}=$ distal and lateral subungual onychomycosis; TDO = total dystrophic onychomycosis.

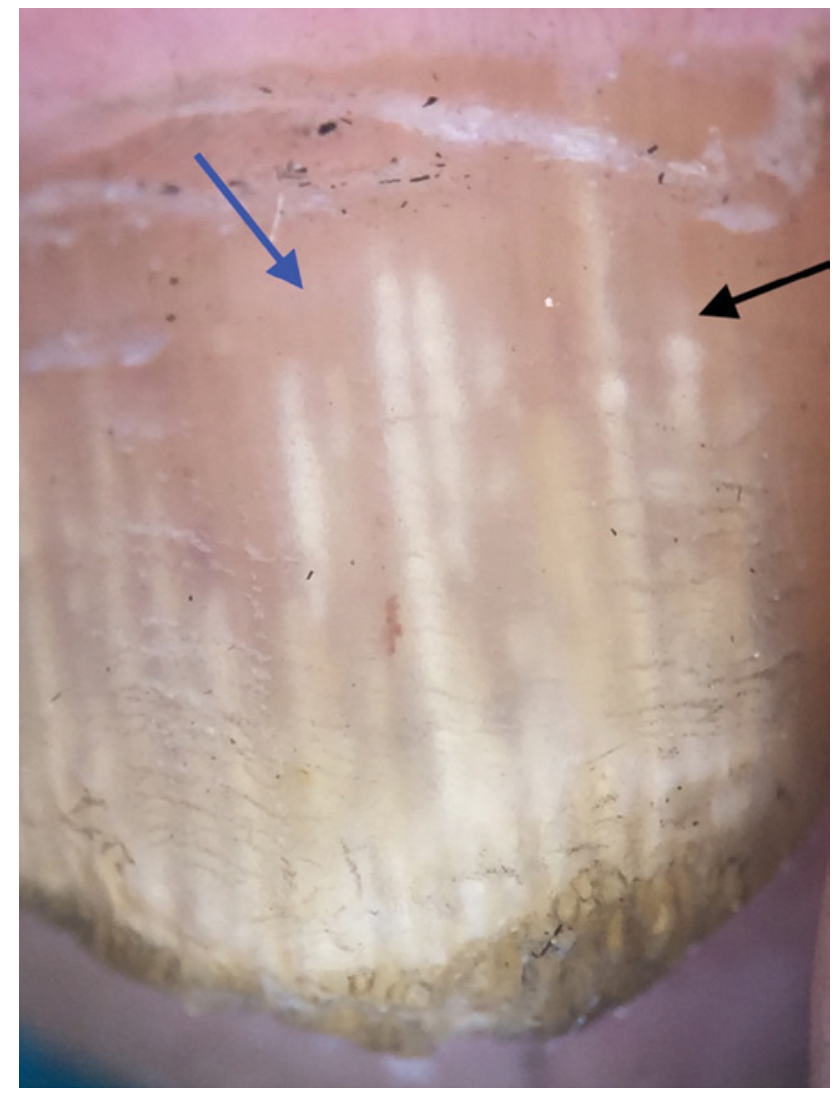

Figure 1. Proximal jagged edge, spikes, and longitudinal striations with different colors (aurora pattern). [Copyright: (O2018 Bhat et al.]

Nail lichen planus, another common disorder, was observed in $27(12 \%)$ patients. Of these, 18 patients were diagnosed on the basis of clinical examination only and 9 patients needed nail biopsy, which showed sawtooth acanthosis, hyperkeratosis, hypergranulosis, degeneration of the basal layer, and bandlike lymphocytic infiltrate. The nail pattern changes that were seen on onychoscopy included chromonychia, subungual hyperkeratosis, onycholysis, and nail plate changes (Table 3; Figures 5 and 6).

Longitudinal melanonychia was seen in $30(13.33 \%)$ patients. Fifteen patients had involvement of a single nail of great toe or thumb, only 6 had involvement of both great toes and thumbs, and 3 had involvement of multiple nails. All patients were subjected to a proper history-taking and

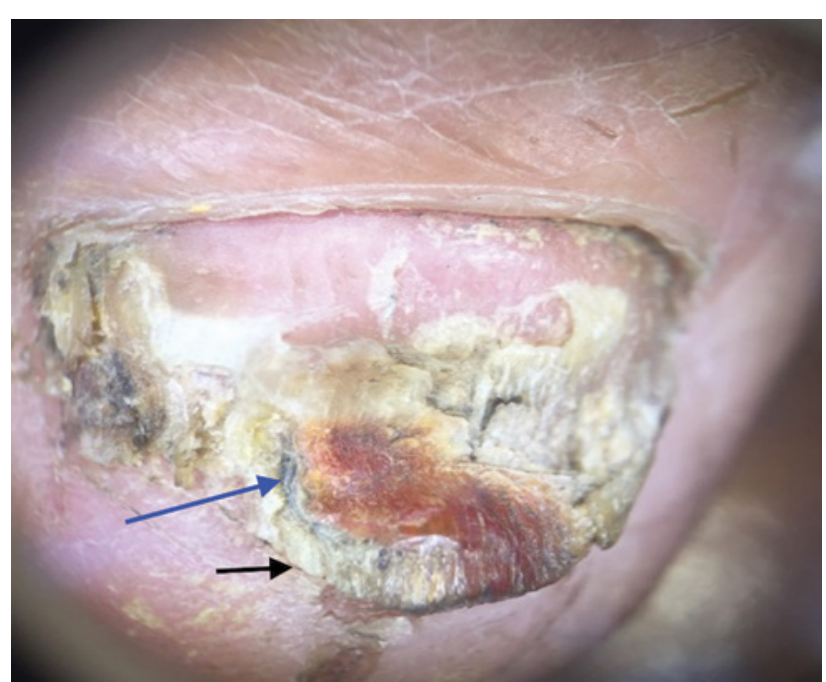

Figure 2. Distal pulverization characteristic of the thickening of the nail plate in total dystrophic onychomycosis. [Copyright: C2018 Bhat et al.]

clinical examination, and in 6 patients nail matrix biopsy was also done. Two patients had features of irregular melanocyte proliferation and their nail biopsy showed features of melanoma, while 4 showed regular proliferation and had benign melanocytic nevus. The other 24 patients had features of melanocytic activation only. Two patients had fungal melanonychia. The onychoscopic changes observed in the benign group were blood spots in 21 patients and regular, parallel, uniform lines in 18 patients. In the melanoma group, irregular lines and micro-Hutchinson sign were seen in both patients (Table 4; Figures 7-9).

Onychoscopic examination of the nail fold capillaries was done in patients with connective tissue disorders by MDAD approach (morphology, diameter, architecture, and density). In this study we examined 24 patients with connective tissue diseases: 11 with systemic sclerosis, 10 with systemic lupus erythematosus (SLE), and 3 with dermatomyositis.

In systemic sclerosis early changes (few enlarged capillaries and few hemorrhages), active disease (frequently enlarged capillaries and frequent hemorrhages), and late changes (irregular enlargement, severe loss of capillaries and avascular areas) were seen. 
TABLE 2. Onychoscopic findings in 63 patients with psoriasis

\begin{tabular}{|c|c|c|}
\hline Onychoscopic Findings & $\begin{array}{l}\text { No. of } \\
\text { Patients }\end{array}$ & Percentage \\
\hline \multicolumn{3}{|l|}{ Nail Matrix Involvement } \\
\hline Pitting & 54 & 85.71 \\
\hline $\begin{array}{l}\text { Ridging (longitudinal or } \\
\text { transverse) }\end{array}$ & 54 & 85.71 \\
\hline Nail plate crumbling & 21 & 33.33 \\
\hline \multicolumn{3}{|l|}{ Nail Bed } \\
\hline $\begin{array}{l}\text { Dilated and tortuous } \\
\text { capillaries in nail bed and } \\
\text { hyponychium }\end{array}$ & 57 & 90.48 \\
\hline Onycholysis & 51 & 80.95 \\
\hline Subungual hyperkeratosis & 27 & 42.86 \\
\hline Salmon patch & 27 & 42.86 \\
\hline Splinter hemorrhages & 15 & 23.81 \\
\hline $\begin{array}{l}\text { Pustules (in pustular } \\
\text { psoriasis) }\end{array}$ & 1 & 1.58 \\
\hline
\end{tabular}

In SLE, we observed capillary dilatations, hemorrhages, telangiectasias, enlarged tortuous capillaries, and normal density. In dermatomyositis, enlarged ramified/bushy capillaries, capillary loss, twisted capillaries, and hemorrhages were seen (Table 5; Figures 10-12).

We found 2 patients with subungual bluish nodules, tender to touch. USG Doppler confirmed the diagnosis of glomus tumor after onychoscopy was done (Figure 13).

\section{Discussion}

The use of dermoscopy in nail disorders is quite recent. Initially its use was limited to nail pigmentations, but nowadays it is frequently being utilized for the diagnosis of other nail disorders as well. In our study, the most common nail disorder observed was onychomycosis and the commonest pattern was distal and lateral subungual onychomycosis. Onychomycosis forms the most common nail disorder and accounts for nearly $50 \%$ of all onychopathies [8].

Since the treatment of onychomycosis can be long-standing and the morphology of the nail changes can vary, diagnosis cannot rely on clinical examination only. Therefore, we use $\mathrm{KOH}$ examination followed by culture of the samples $[9,10]$. Because culture is a time-consuming process and to decrease the number of samples to be sent for culture, we can utilize a dermatoscope, which is a noninvasive procedure that can aid in the diagnosis of onychomycosis.

Piraccini et al [11] retrospectively analyzed 57 digital dermoscopic images with clinical diagnosis of onycholysis; they identified and described specific dermoscopic signs for onychomycosis. Dermoscopic findings described for ony-

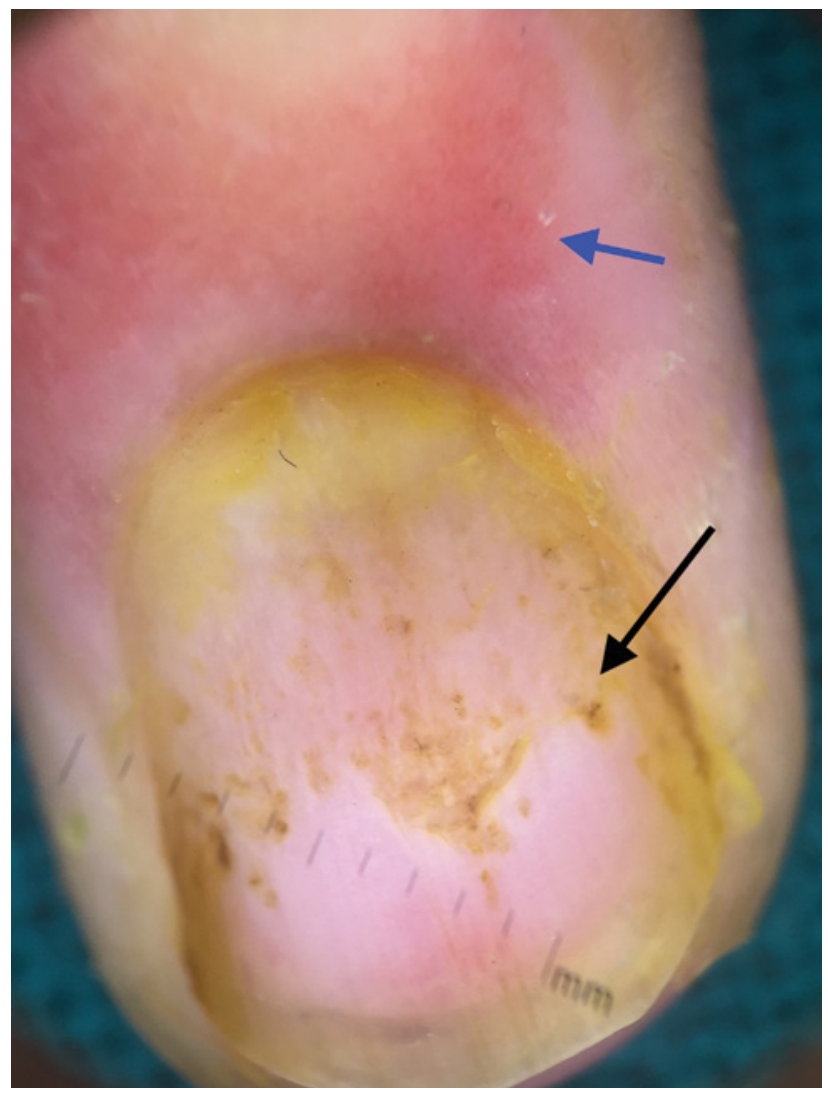

Figure 3. Coarse pits irregular in size and shape, yellowish discoloration, distal onycholysis, capillary changes in proximal nail fold. [Copyright: @2018 Bhat et al.]

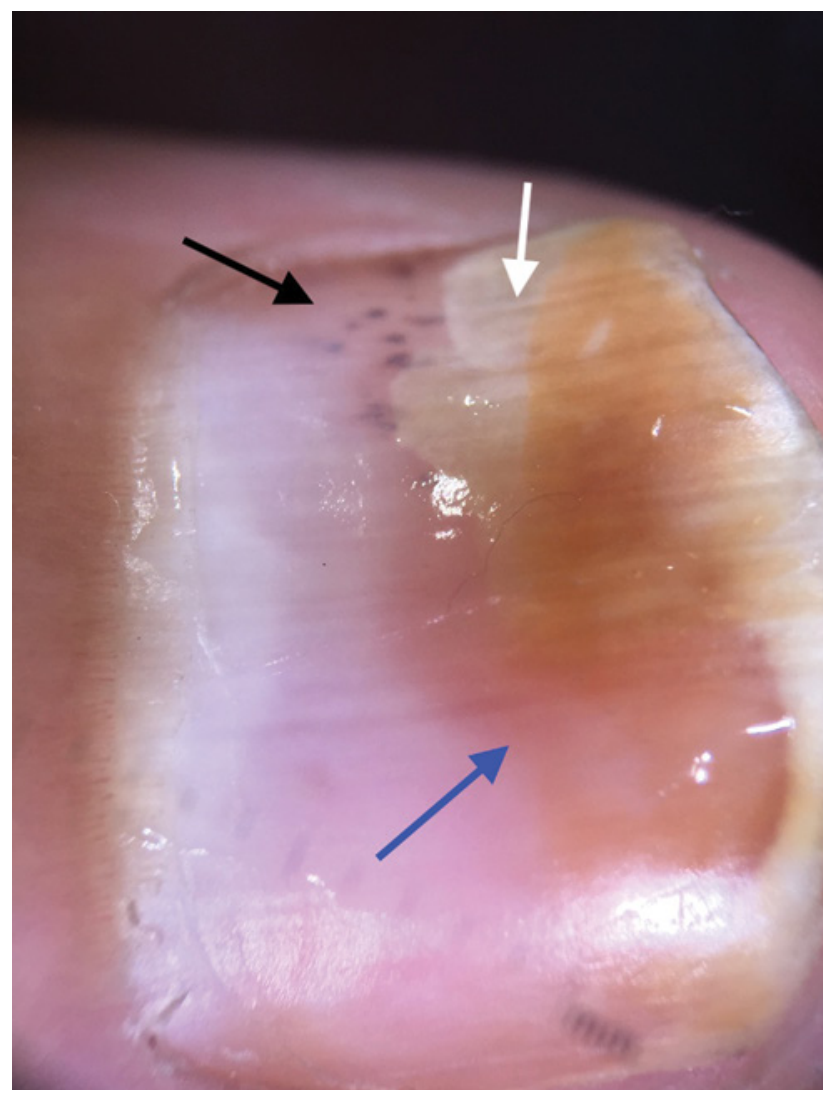

Figure 4. Distal onycholysis with proximal regular and erythematous border, salmon spot (orange to red), and splinter hemorrhages (purplish linear streaks). [Copyright: (02018 Bhat et al.] 
TABLE 3. Onychoscopic findings in 27 patients with nail lichen planus

\begin{tabular}{|c|c|c|}
\hline Onychoscopic Findings & $\begin{array}{c}\text { No. of } \\
\text { Patients }\end{array}$ & Percentage \\
\hline \multicolumn{3}{|l|}{ Nail Matrix } \\
\hline Longitudinal streaks & 24 & 88.89 \\
\hline Pitting & 12 & 44.44 \\
\hline Anonychia & 12 & 44.44 \\
\hline Pterygium & 9 & 33.33 \\
\hline Red lunula & 6 & 22.22 \\
\hline Trachyonychia & 4 & 14.81 \\
\hline \multicolumn{3}{|l|}{ Nail Bed } \\
\hline Nail fragmentation & 24 & 88.89 \\
\hline Chromonychia & 18 & 66.67 \\
\hline Onycholysis & 9 & 33.33 \\
\hline Subungual hyperkeratosis & 6 & 22.22 \\
\hline Splinter hemorrhages & 3 & 11.11 \\
\hline
\end{tabular}

chomycosis were jagged proximal edge with spikes of the onycholytic area and longitudinal striae [11]. Nakamura et al [8] performed dermoscopy in 500 cases of nail disorders, and in onychomycosis they identified chromonychia, onycholysis, opacity, and longitudinal stripes.

In our study, the dermoscopic findings observed in onychomycosis showed jagged pattern of the proximal margin of the onycholytic area, spikes that were directed to the proximal fold, white-yellow longitudinal striae in the onycholytic nail plate (aurora borealis pattern), and distal irregular termination pattern.

The next common onychopathy in our study was nail psoriasis. Psoriasis can involve any structure of the nail apparatus, and accordingly there are different clinical manifestations [12]. So far the dermoscopic findings that have been observed in nail psoriasis include pitting, onycholysis, salmon spot, dilated blood vessels, splinter hemorrhages, dilated and tortuous capillaries in the hyponychium, and subungual hyperkeratosis $[13,14]$. Fine pits could be appreciated well with a dermatoscope[15].

We divided the nail unit changes in our patients into nail matrix and nail bed changes. The nail matrix changes include pitting, both longitudinal and transverse ridging, and nail plate crumbling. The nail bed changes are dilation and tortuosity of capillaries, onycholysis, subungual hyperkeratosis, salmon patch, and splinter hemorrhages.

Dermoscopy is also a complementary tool in the diagnosis of nail lichen planus. Since nail lichen planus affects both nail matrix and nail bed, the clinical signs also vary. Dermoscopy findings that have been observed with matrix involvement include fissuring, pitting, trachyonychia, longitudinal ridging, dorsal pterygium, erythematous patches in the lunula, mela-

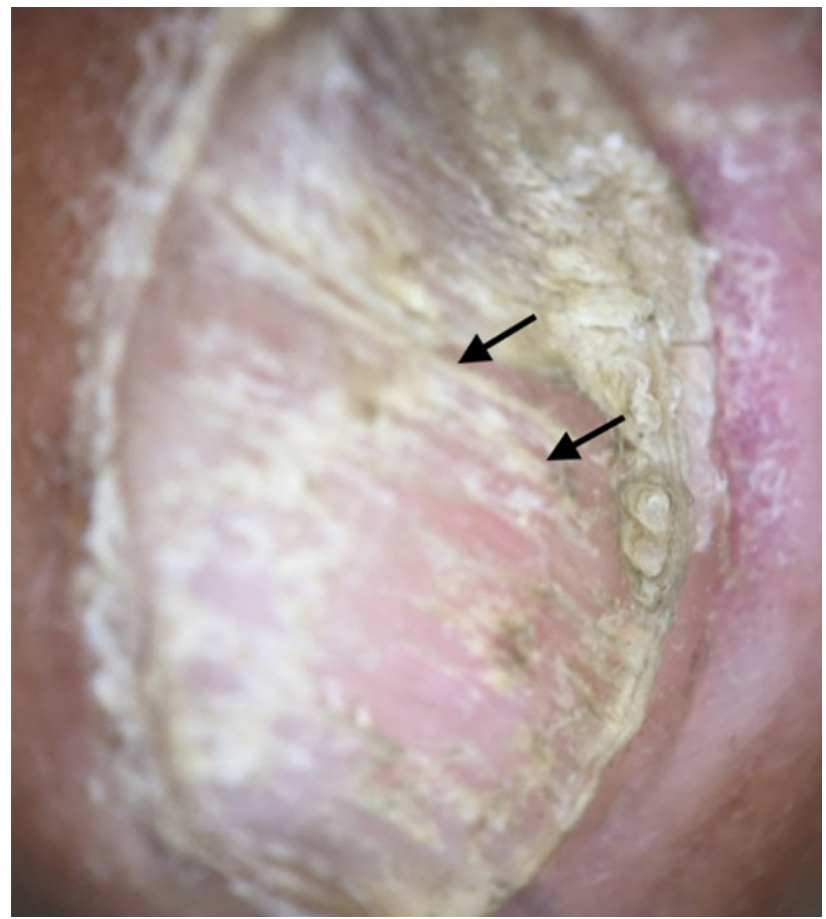

Figure 5. Longitudinal fissures, fragmentation, thinning of nail plate. [Copyright: (O2018 Bhat et al.]

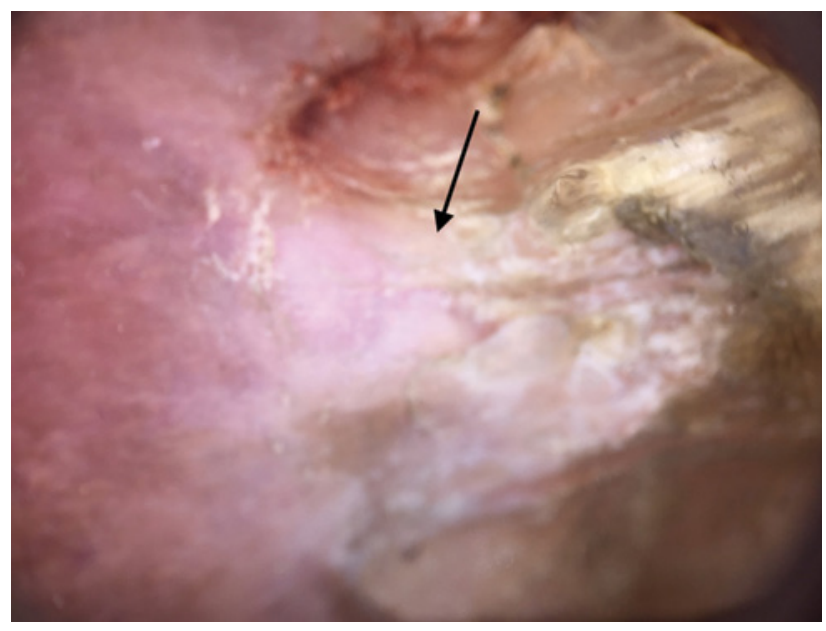

Figure 6. Dorsal pterygium formation. [Copyright: (2)18 Bhat et al.]

nonychia, and atrophy; nail bed involvement shows subungual hyperkeratosis, chromonychia, and onycholysis [16,17]. In our experience with biopsy-confirmed nail lichen planus, the observed dermoscopic findings were chromonychia, whitish striations, nail fragmentation, trachonychia, onycholysis, and nail plate destruction; in clinically suspected cases, the observed dermoscopic findings were trachonychia, pitting, anonychia, pterygium, red lunula, chromonychia, subungual hyperkeratosis, onycholysis, striations, nail fragmentation, splinter hemorrhages, and nail plate destruction.

Another nail change that can be of concern in most patients is longitudinal melanonychia. This may represent a benign melanocytic nevus, lentigo, racial/ethnic mela- 
TABLE 4. Onychoscopic findings in longitudinal melanonychia

\begin{tabular}{|l|c|c|c|c|c|c|c|c|} 
& \multicolumn{2}{|c|}{ Blood Spots } & \multicolumn{2}{c|}{ Regular Lines } & \multicolumn{2}{c|}{ Irregular Lines } & \multicolumn{2}{c|}{ Micro-Hutchinson Sign } \\
\hline & No. & $\%$ & No. & $\%$ & No. & $\%$ & No. & $\%$ \\
\hline Total & 21 & 70 & 18 & 60 & 2 & 6.67 & 2 & 6.67 \\
\hline
\end{tabular}

$\mathrm{P}>0.05$; chi-square statistic $=0.083$.

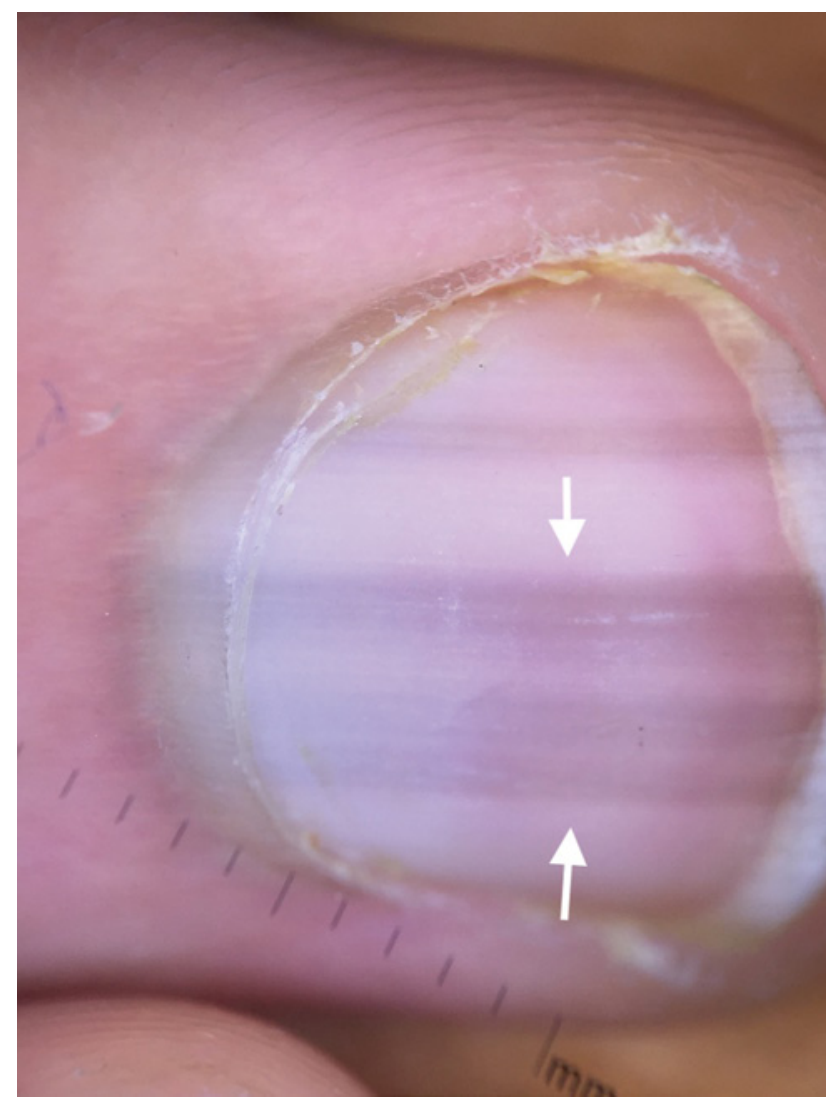

Figure 7. Longitudinal melanonychia due to melanocytic activation with regular, parallel, and uniform gray bands. [Copyright: C2018 Bhat et al.]

nonychia, drug-induced hyperpigmentation, or at times a malignant melanoma. The most common site involved in melanonychia is the thumb, followed by the great toe and the index finger [18].

To distinguish benign causes of longitudinal melanonychia from malignant ones, dermoscopy appears to be helpful to clinicians. It allows the visualization and evaluation of morphological features that are otherwise not visible to the unaided eye and thus forms a link between macroscopic clinical dermatology and microscopic dermatopathology [19]. In a study conducted by Ronger et al [20], investigators observed 7 dermoscopic patterns in longitudinal melanonychia: (1) blood spots, (2) brown coloration of the background, (3) regular lines,(4) irregular lines, (5) grayish background and thin gray lines, (6) micro-Hutchinson sign, and (7) microscopic grooves. Among these patterns, brown-black background hue, irregular longitudinal lines, and micro-Hutchinson sign

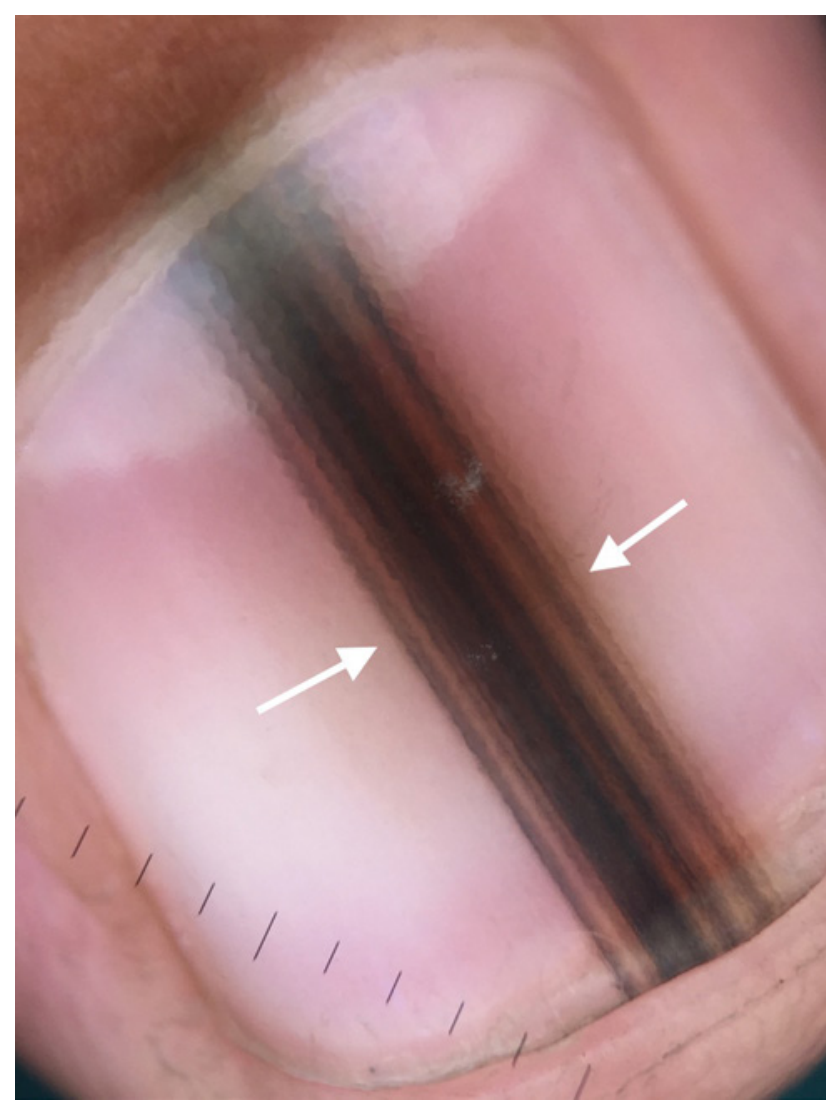

Figure 8. Longitudinal melanonychia due to benign melanocytic nevus showing regular, parallel, and uniform brown-black bands with negative Hutchinson sign. [Copyright: @2018 Bhat et al.]

are more often seen in malignant melanoma [20]. In this study we observed blood spots and regular lines in patients with benign cause and irregular lines and micro-Hutchinson sign in patients with malignant melanoma.

Proximal nail fold changes are also common in connective tissue diseases. Although most nail fold changes in connective tissue diseases are not specific, they may give an important clue to the diagnosis. A dermatoscope can help to detect these findings, which are difficult to see with the unaided eye. A case-control study compared nail changes in patients with connective tissue diseases and 2 healthy groups [21]. In this study, capillary loops and splinter hemorrhages were frequent significantly in patients with scleroderma. Other studies have shown an association between the degree of nail fold capillary abnormalities and internal organ involvement and mortality in patients with scleroderma $[22,23]$. In our study the onychoscopic findings observed 


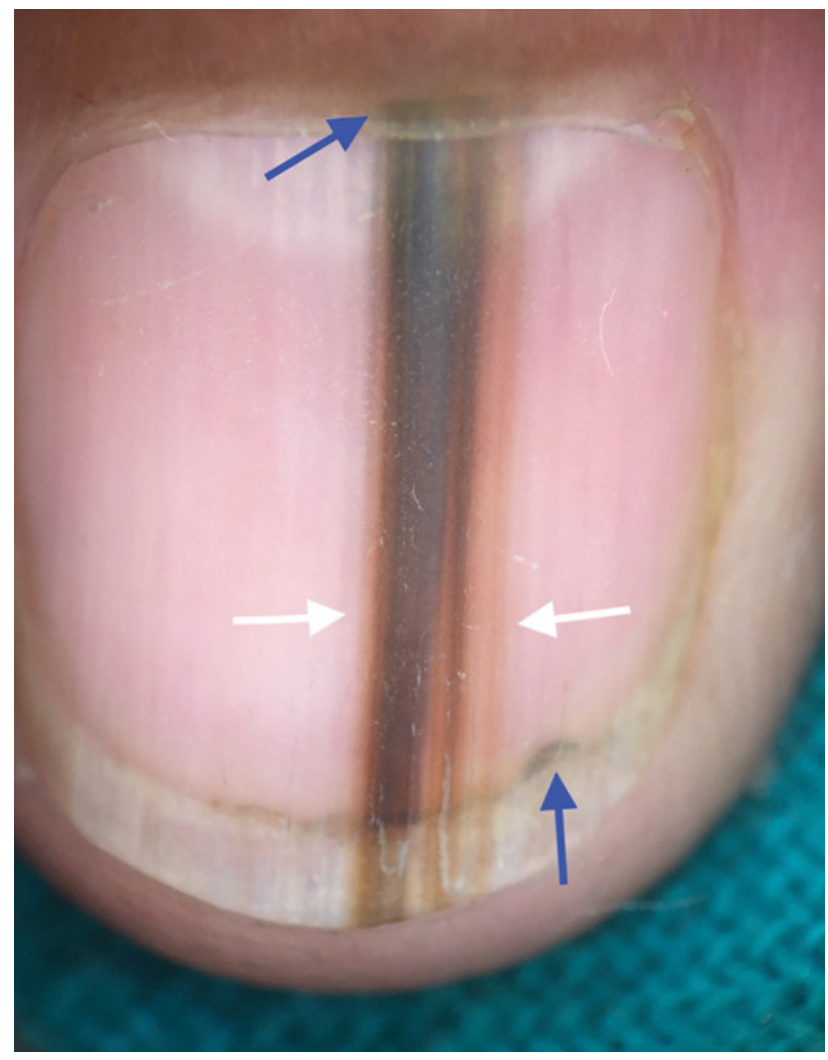

Figure 9. Longitudinal melanonychia due to melanoma showing irregular black-gray-brown bands which are not parallel and are not uniform in color. Micro-Hutchinson's sign is positive. [Copyright: (C)2018 Bhat et al.]

in patients with systemic sclerosis were enlarged capillaries, loss of capillaries, avascular areas, hemorrhages, and irregular enlargement.
Various studies have investigated nail changes in dermatomyositis; nail changes were observed in the form of splinter hemorrhages, red lunula, cuticular changes, and capillary loops in proximal nail folds [24-26]; in our study we noticed enlarged and ramified capillaries, capillary loss, twisted capillaries, and hemorrhages. Also in previous studies, nail changes observed in patients with SLE were proximal nail fold erythema, longitudinal ridging, splinter hemorrhages, bluish-black discoloration of the nail plate, onycholysis, subungual hyperkeratosis, and red lunula. Splinter hemorrhage in fingernails of patients with SLE was found to be associated significantly with disease activity [27]. We found nail changes in the form of capillary dilations and enlarged tortuous capillaries with normal density.

Glomus tumors are painful benign hamartomas with increased sensitivity to cold that arise from myoarterial apparatus [28,29]. We found 2 such patients, who showed pinkish blush in the nail plate with onychoscopy.

\section{Limitations}

Onychoscopy can be used as a diagnostic tool for nail disorders; however, it cannot replace the histopathological examination when there is a diagnostic dilemma. In our study, the sample size for various disorders was too small to allow interpretation that onychoscopy can be used as a diagnostic tool for those disorders. To validate these findings, therefore, further studies with larger sample sizes are needed.

TABLE 5. Onychoscopic findings in patients with connective tissue disorders Disease Onychoscopic Findings

No. of Patients

Percentage

\begin{tabular}{|c|c|c|c|}
\hline \multicolumn{4}{|l|}{ Systemic Sclerosis } \\
\hline \multirow{4}{*}{ Nail fold changes } & Enlarged capillaries & $8 / 11$ & 72.73 \\
\hline & Irregular enlargement of the capillaries & $7 / 11$ & 63.64 \\
\hline & Loss of capillaries & $6 / 11$ & 54.54 \\
\hline & Avascular areas & $6 / 11$ & 54.54 \\
\hline Nail bed changes & Hemorrhages & $5 / 11$ & 45.45 \\
\hline \multicolumn{4}{|c|}{ Systemic Lupus Erythematosus } \\
\hline \multirow{3}{*}{ Nail fold changes } & Capillary dilations & $8 / 10$ & 80 \\
\hline & Enlarged tortuous capillaries & $8 / 10$ & 80 \\
\hline & Periungual telangiectasia & $6 / 10$ & 60 \\
\hline Nail bed changes & Hemorrhages & $4 / 10$ & 40 \\
\hline \multicolumn{4}{|l|}{ Dermatomyositis } \\
\hline \multirow{3}{*}{ Nail fold changes } & Enlarged capillaries & $3 / 3$ & 100 \\
\hline & Capillary loss & $2 / 3$ & 66.67 \\
\hline & Twisted capillaries & $2 / 3$ & 66.67 \\
\hline Nail bed changes & Hemorrhages & $2 / 3$ & 66.67 \\
\hline
\end{tabular}




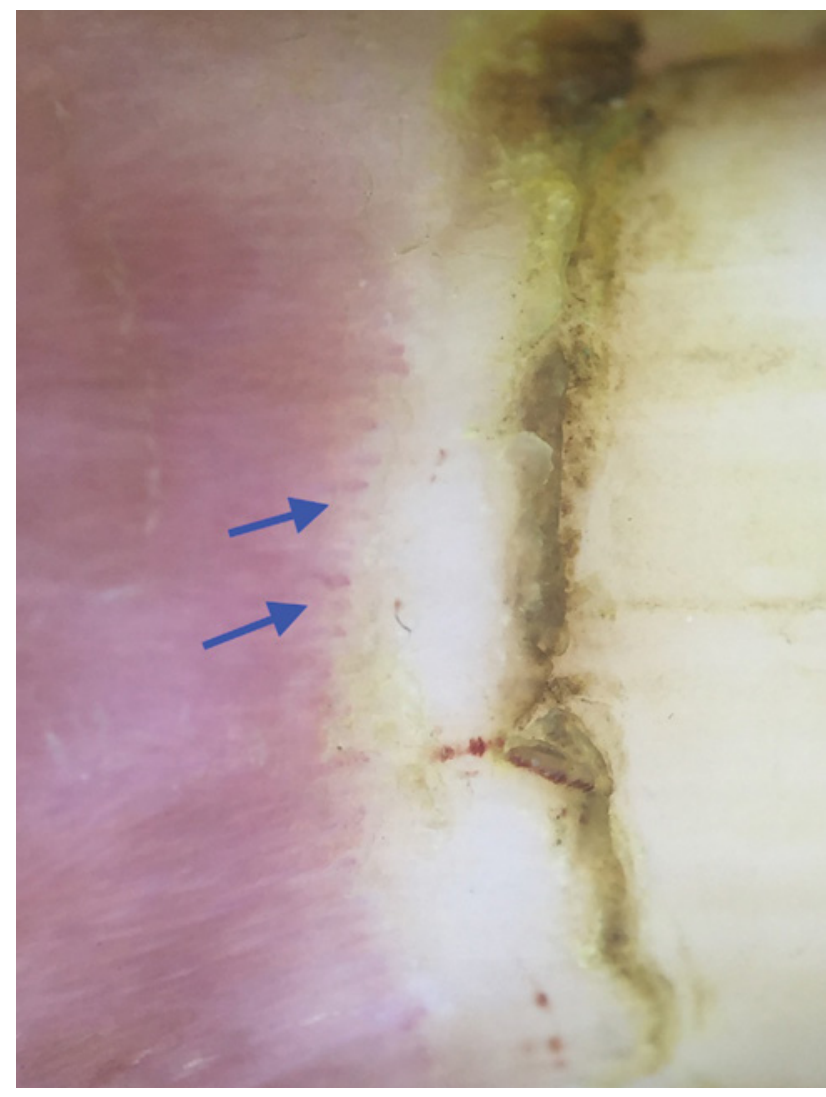

Figure 10. Proximal nail fold capillaries in systemic sclerosis: enlarged capillaries, cuticular hemorrhages, and loss of capillaries leading to avascular areas. [Copyright: (02018 Bhat et al.]

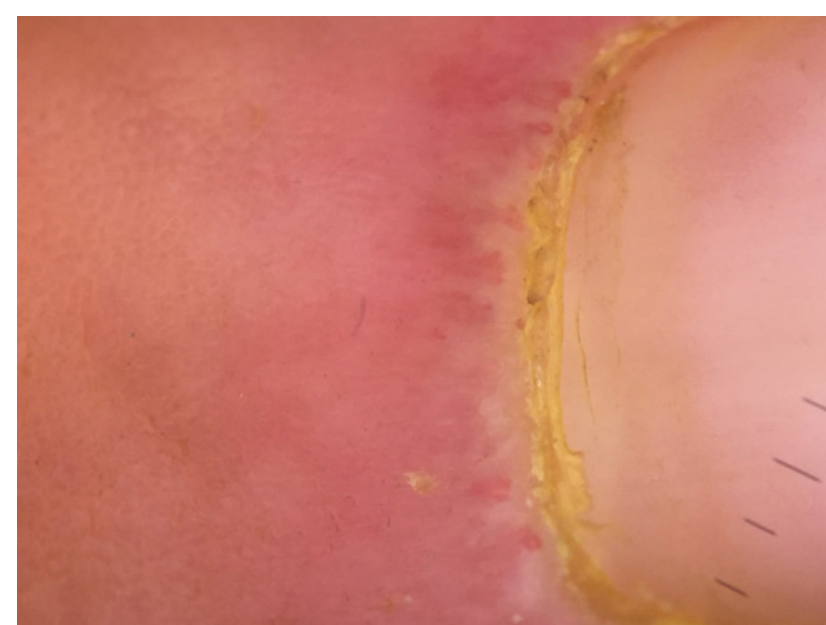

Figure 12. Proximal nail fold capillaries in dermatomyositis: enlarged ramified/bushy capillaries with capillary loss. [Copyright: C)2018 Bhat et al.]

\section{Conclusions}

Onychoscopy is an easier, noninvasive, and cost-effective diagnostic tool that can allow detection of subtle nail changes not visible to the unaided eye. It can aid in diagnosing nail disorders earlier so that we can treat them before the disease progresses. In addition, it can help in differentiating benign lesions from malignant ones and accordingly guide us to

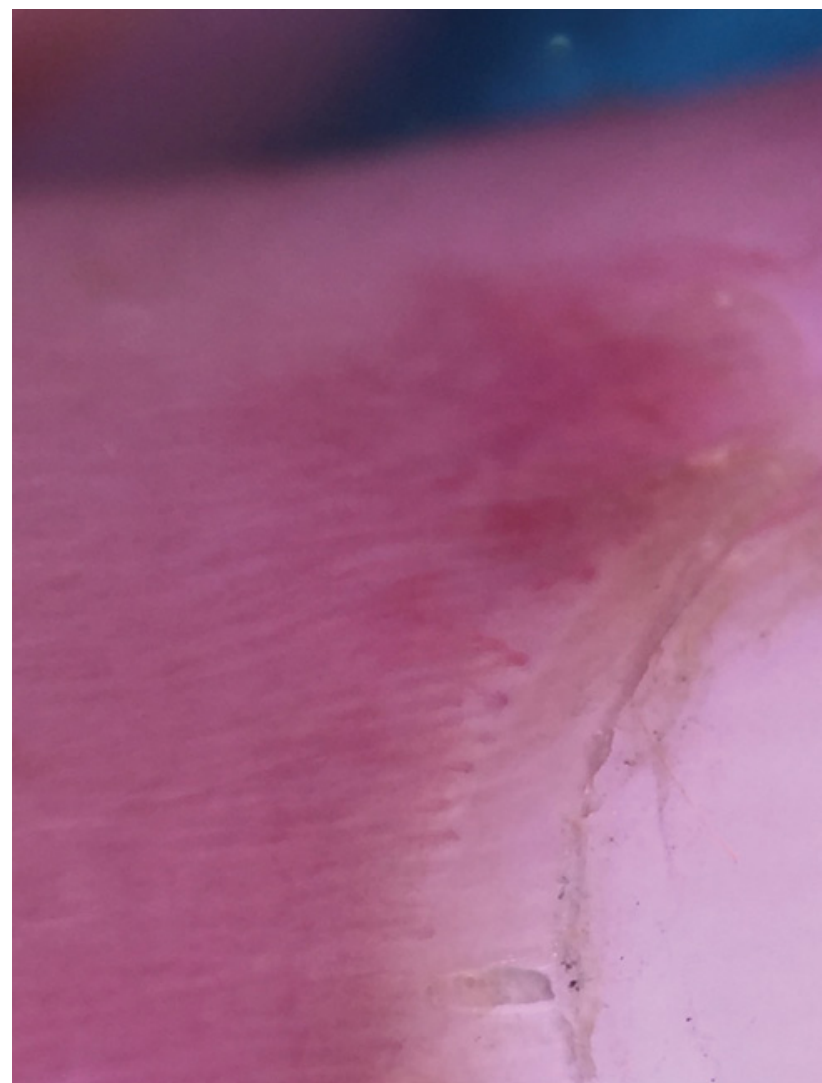

Figure 11. Proximal nail fold capillaries in SLE: enlarged tortuous capillaries and normal density. [Copyright: @2018 Bhat et al.]

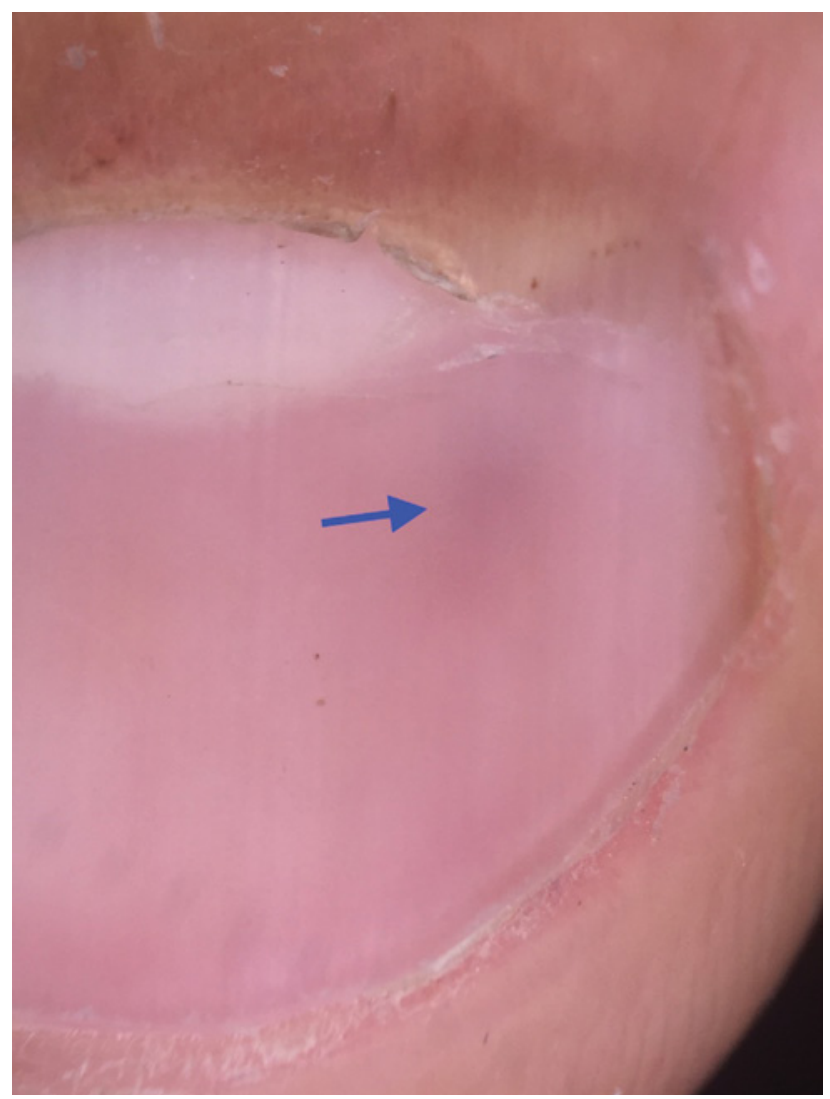

Figure 13. Glomus tumor presenting as pinkish vascular area in nail bed. [Copyright: @2018 Bhat et al.] 
avoid unnecessary biopsies. However, the interpreter needs to have a thorough knowledge of the nail anatomy and its disorders; onychoscopy should not be used as the only diagnostic tool for confirming the diagnosis.

\section{References}

1. Samman PD. The Nail in Disease. 2nd ed. London: Heineman W; 1972:1-176.

2. Campos-do-Carmo G, Ramos-e-Silva M. Dermoscopy: basic concepts. Int J Dermatol. 2008;47(7):712-719.

3. Koga H, Saida T, Uhara H. Key point in dermoscopic differentiation between early nail apparatus melanoma and benign longitudinal melanonychia. J Dermatol. 2011;38(1):45-52.

4. Ronger S, Touzet S, Ligeron C, et al. Dermoscopic examination of nail pigmentation. Arch Dermatol. 2002;138(10):1327-1333.

5. Braun RP, Baran R, Le Gal FA, et al. Diagnosis and management of nail pigmentations. J Am Acad Dermatol. 2007;56(5):835-847.

6. Jellinek N.Nail matrix biopsy of longitudinal melanonychia: diagnostic algorithm including the matrix shave biopsy. $\mathrm{J} \mathrm{Am} \mathrm{Acad}$ Dermatol. 2007;56(5):803-810.

7. Ronger S, Touzet S, Ligeron C, et al. Dermoscopic examination of nail pigmentation. Arch Dermatol. 2002;138(10):1327-1333.

8. Nakamura RC, Costa M. Dermatoscopic findings in the most frequent onychopathies: descriptive analysis of 500 cases. Int $J$ Dermatol. 2012;51(4):483-496.

9. Richert B, Lateur N, Theunis A, et al. New tools in nail disorders. Semin Cutan Med Surg. 2009;28(1):44-48.

10. Harvey CK, Richardson A. Techniques for obtaining specimens for culture to confirm onychomycosis. J Am Podiatr Med Assoc. 2000;90(8):394-396.

11. Piraccini BM, Balestri R, Starance M, et al. Nail digital dermoscopy (onychoscopy) in the diagnosis of onychomycosis. J Eur Acad Dermatol Venereol. 2013;27(4):509-513.

12. Sánchez-Regaña M, Umbert P. Diagnosis and management of nail psoriasis [in Spanish]. Actas Dermosifiliogr. 2008;99(1):34-43.

13. Piraccini BM, Bruni F, Starace M. Dermoscopy of non-skin cancer nail disorders. Dermatol Ther. 2012;25(6):594-602.

14. Iorizzo M, Dahdah M, Vincenzi C, et al. Videodermoscopy of the hyponychium in nail bed psoriasis. J Am Acad Dermatol. 2008;58(4):714-715.
15. Farias DC, Tosti A, Chiacchio ND, Hirata, SH. Dermoscopy in nail psoriasis [in Portuguese]. An Bras Dermatol. 2010;85(1):101103.

16. Nakamura R, Broce AA, Palencia DP, Ortiz NI, Leverone A. Dermatoscopy of nail lichen planus. Int J Dermatol.2013;52(6):684687.

17. Goettmann S, Zaraa I, Moulonguet I. Nail lichen planus: epidemiological, clinical, pathological, therapeutic and prognosis study of 67 cases. J Euro Acad Dermatol Venereol. 2012;26(10):13041309.

18. Koga H, Saida T, Uhara H. Key point in dermoscopic differentiation between early nail apparatus melanoma and benign longitudinal melanonychia. J Dermatol. 2011;38(1):45-52.

19. Argenziano G, Soyer HP. Dermoscopy of pigmented skin lesionsa valuable tool for early diagnosis of melanoma. Lancet Oncol. 2001;2(7):443-449.

20. Ronger S, Touzet S, Ligeron C, et al. Dermoscopic examination of nail pigmentation. Arch Dermatol. 2002;138(10):1327-1333.

21. Nabil PA, Rao R, Shenoi SD, Balachandran C. Nail unit in collagen vascular diseases: a clinical, histopathological and direct immunofluorescence study. Indian J Dermatol. 2006;51(4):265268.

22. Ingegnoli F, Ardoino I, Boracchi P, Cutolo M; EUSTAR coauthors. Nailfold capillaroscopy in systemic sclerosis: data from the EULAR scleroderma trials and research (EUSTAR) database. Microvasc Res. 2013;89:122-128.

23. Smith V, Decuman S, Sulli A, et al. Do worsening scleroderma capillaroscopic patterns predict future severe organ involvement? A pilot study. Ann Rheum Dis. 2012;71(10):1636-1639.

24. Dourmishev LA, Dourmishev AL. Dermatomyositis: Advances in Recognition, Understanding and Management. Berlin, Heidelberg: Springer-Verlag; 2009.

25. Samitz MH. Cuticular changes in dermatomyositis. Arch Dermatol. 1974;110(6):866-867.

26. Tosti A, De Padova MP, Fanti P, et al. Unusual severe nail involvement in dermatomyositis. Cutis. 1987;40(3):261-262.

27. Ekmekc Tr, Ucak S, Aslan K, et al. Exaggerated cuticular changes in a patient with dermatomyositis. J Euro Acad Dermatol Venereol. 2005;19(1):135-136.

28. McDermott EM, Weiss AP. Glomus tumors. J Hand Surg Am. 2006;31(8):1397-1400.

29. Baran R, Richert B. Common nail tumors. Dermatol Clin. 2006;24(3):297-311. 